\title{
Summary of the Blind Test Campaign to predict the High Reynolds number performance of DU00-W-210 airfoil
}

\author{
Özlem Ceyhan Yilmaz \\ ECN, Energy Research Center of the Netherlands, Petten, the Netherlands \\ Oscar Pires and Xabier Munduate \\ CENER, National Renewable Energy Centre, Sarriguren, Spain \\ N. Sørensen \\ DTU, Frederiksborgvej 399, 4000, Roskilde, Denmark \\ Torben Reichstein and A. P. Schaffarczyk \\ Kiel University of Applied Sciences, Kiel, Germany \\ Konstantinos Diakakis and Giorgos Papadakis \\ NTUA, National Technical University of Athens, Athens, Greece \\ Elia Daniele \\ Fraunhofer IWES, Kupkersweg 70, 26129 Oldenburg, Germany \\ Michael Schwarz \\ Forwind, Kupkersweg 70, 26129 Oldenburg, Germany \\ Thorsten Lutz \\ University of Stuttgart, Pfaffenwaldring 21, 70569 Stuttgart, Germany \\ Raul Prieto \\ Offshore Renewable Energy Catapult, Albert Street NE24 ILZ, Blyth, UK
}

\begin{abstract}
This paper summarizes the results of a blind test campaign organized in the AVATAR project to predict the high Reynolds number performance of a wind turbine airfoil for wind turbine applications. The DU00-W-210 airfoil was tested in the DNW-HDG pressurized wind tunnel in order to investigate the flow at high Reynolds number range from 3 to 15 million which is the operating condition of the future large $10 \mathrm{MW}+$ offshore wind turbine rotors. The results of the experiment was used in a blind test campaign to test the prediction capability of the CFD tools used in the wind turbine rotor simulations. As a result of the blind test campaign it was found that although the codes are in general capable of predicting increased max lift and decreased minimum drag with Re number, the Re trend predictions in particular the glide ratio (lift over drag) need further improvement. In addition to that, the significant effect of the inflow turbulence on glide ratio especially at high Re numbers is found as the most important parameter where the prediction as well as the selection of the correct inflow turbulence levels is the key for correct airfoil designs for the future generation $10 \mathrm{MW}+$ wind turbine blades.
\end{abstract}

\section{Introduction}

$\mathrm{N}$ ext generation large offshore wind turbines of 10MW+ range bring new questions regarding the reliability and the accuracy of the design codes used by the wind energy community. EU FP7 project AVATAR (AdVanced Aerodynamic Tools of 1Arge Rotors) addresses the challenges in aerodynamic modelling of 10MW+ wind turbines as the aerodynamic codes are not yet validated for these large machines. One of the most obvious change going from 
conventional wind turbine size to the $10 \mathrm{MW}+$ range is the increase in Re number to the range that the wind energy society has never entered before[21]. In order to investigate the effects of the increase in the Reynolds numbers, an airfoil is tested in a pressured wind tunnel of DNW in Gottingen. Since this wind tunnel is pressurized, it is possible to reach high Reynolds numbers without suffering from the compressibility effects of increased Mach numbers, as a result, it is possible to investigate isolated Reynolds number effects. Detailed and high quality test results are obtained for a range of Re numbers by acquiring the data from the surface pressures, wake rake, balance, hot wire probe at the entrance of the test section and the flow visualization. Before the results of the experiment was shared within the project, a blind test campaign was organized to compare the predictions from different CFD codes of the tested conditions. A number of cases were selected and put in a document together with all the information necessary to run the simulations[22]. The results were presented to the participants during the AVATAR workshop in EWEA Paris 2015. In this paper, the results of this blind test campaign will be summarized.

\section{Experimental Data}

\section{A. Wind Tunnel, Model and Instrumentation}

DNW-HDG wind tunnel is a closed circuit wind tunnel as shown in Figure 1 with a test section of $60 \mathrm{~cm} \times 60 \mathrm{~cm}$. It is powered with an external motor and connected to a fan with an rpm range of 200 to 820 with a fixed fan blade angle. The tunnel can be pressurized from 1 to $100 \times 10^{5} \mathrm{~Pa}$ where the tunnel temperature is changing from ambient to 45 degrees. The velocity and pressure range that this tunnel can reach to obtain different Re numbers is plotted in Figure 2.

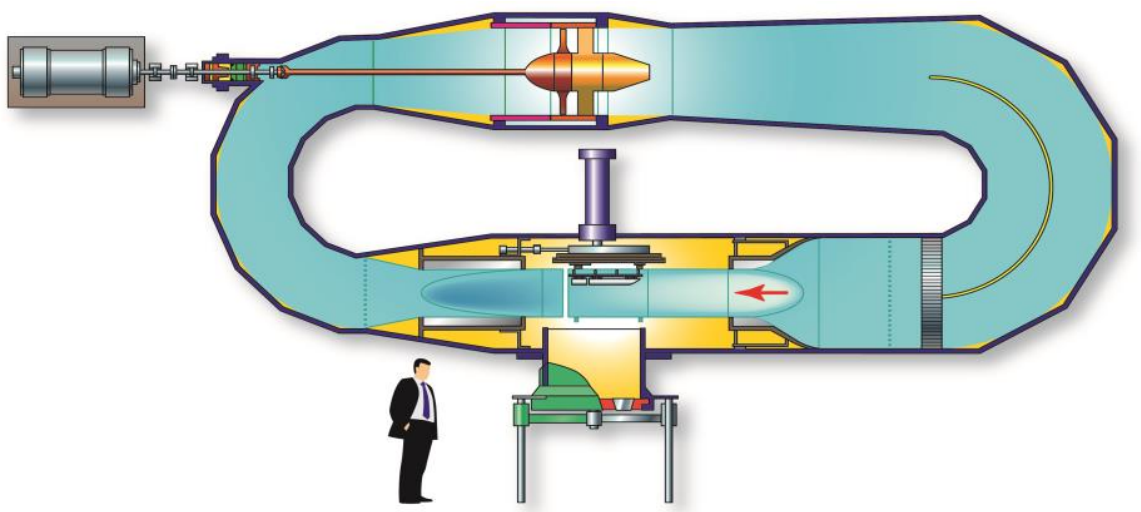

Figure 1. DNW-HDG wind tunnel

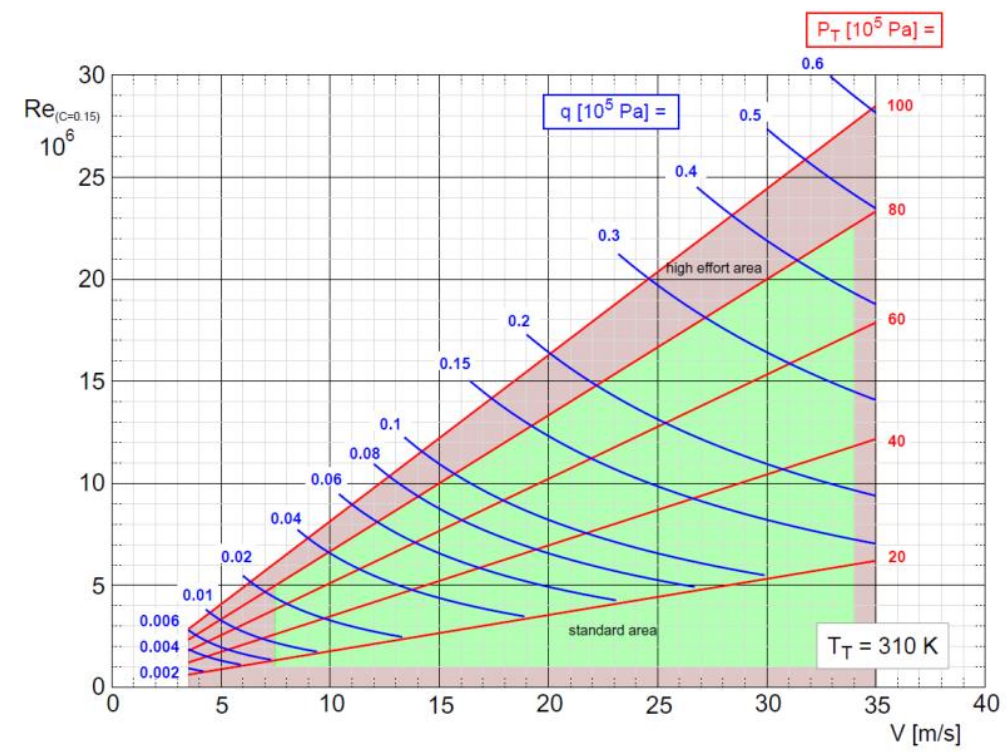

Figure 2. Tunnel pressure and speeds to obtain different Reynolds numbers

American Institute of Aeronautics and Astronautics 
The airfoil used in this experiment is $21 \%$ thick DU00-W-212 airfoil which is developed in the Delft University of Technology in the Netherlands. The wind tunnel model of this airfoil has $150 \mathrm{~mm}$ chord length and $600 \mathrm{~mm}$ span and it is made of aluminum as shown inside the test section in Figure 3. A special surface treatment is applied on the surface finish of this model in order to keep a surface roughness of $0.03 \mu \mathrm{m}$ in terms of $\mathrm{Rz}$ (the mean roughness depth). The model is equipped with 90 pressure taps which is located on the half span and used for the lift measurements. They are positioned with an angle to the span in order to reduce the interference of the taps to the flow. Drag is measured with a wake rake which is able to traverse half-span. In addition to those, a probe holder is located at the entrance of the test section with a hot wire, five Kulite pressure sensors, and three component balance at the sides of the wall were also used during the experiment.

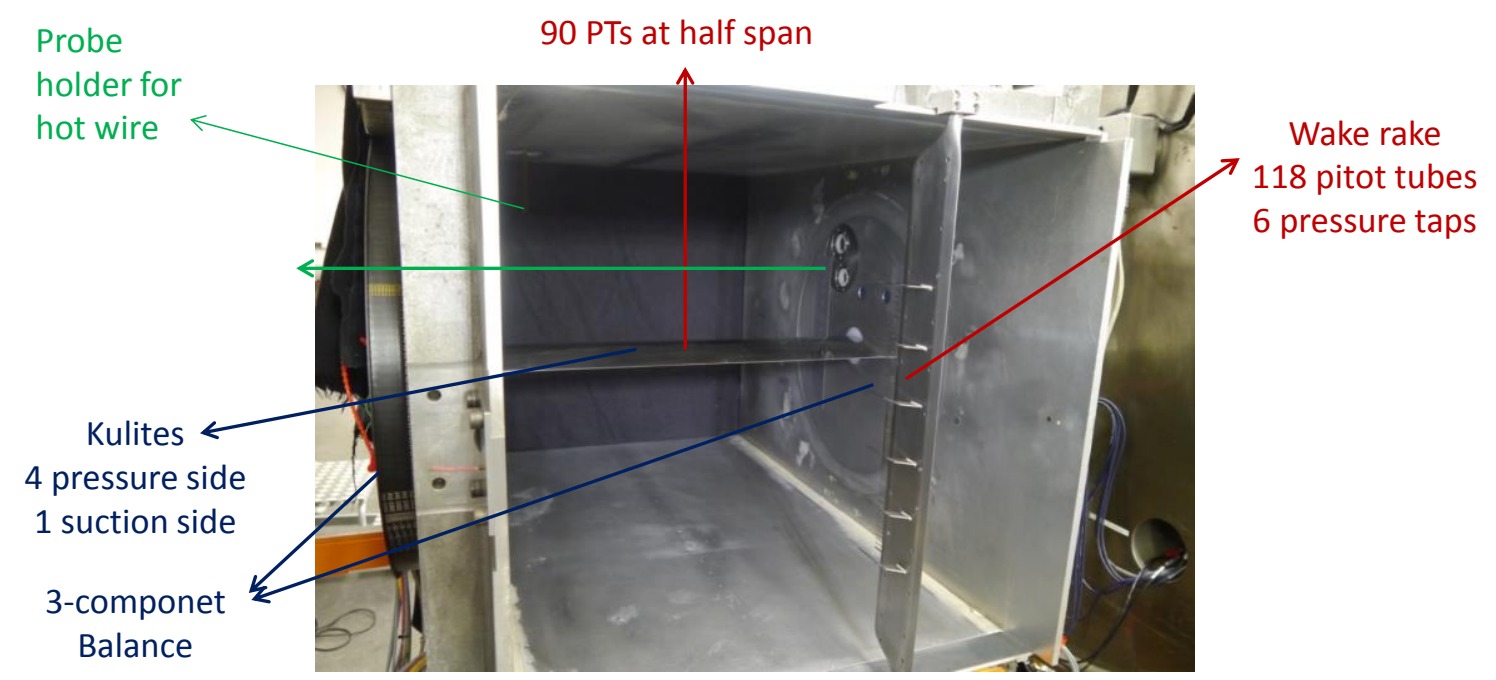

Figure 3. Test section equipped with DU00-W-210 airfoil model and instrumentation.

\section{B. Test Cases used in the Blind test study}

Among all the conditions that are tested in the wind tunnel, seven of them are chosen for the blind test campaign. These cases are summarized in Table 1. For these cases, the turbulence intensity is used in order to specify the tunnel turbulence level to be used in the computations. This turbulence intensity is calculated from the predicted $\mathrm{N}$ factors using the empirical relation $\mathrm{N}=-8.43-2.4 \ln (\mathrm{T})$ as recommended in [24] where the $\mathrm{N}$ factors are calculated using XFOIL and RFOIL. Although most of the participating codes use the $\mathrm{e}^{\mathrm{N}}$ transition model, the predictions were expected to have an uncertainty coming from the way how the tunnel turbulence is specified for the simulations.

\begin{tabular}{|c|c|c|c|c|c|c|c|}
\hline & $\begin{array}{c}\text { Test } 1 . \\
R e=3 \mathrm{mil}\end{array}$ & $\begin{array}{c}\text { Test } 2 . \\
\text { Re=6mil-1 }\end{array}$ & $\begin{array}{c}\text { Test } 3 . \\
\text { Re=6mil-2 }\end{array}$ & $\begin{array}{c}\text { Test } 4 . \\
\text { Re=9mil-1 }\end{array}$ & $\begin{array}{c}\text { Test } 5 \\
\text { Re=9mil-2 }\end{array}$ & $\begin{array}{c}\text { Test } 6 . \\
R e=12 \mathrm{mil}\end{array}$ & $\begin{array}{c}\text { Test } 7 . \\
\text { Re=15mil }\end{array}$ \\
\hline$P_{t}[$ bars $]$ & 12 & 34 & 67 & 34 & 67 & 67 & 60 \\
\hline $\mathrm{V}_{\text {tunnel }}[\mathrm{m} / \mathrm{s}]$ & 25.6 & 19 & 10 & 28.5 & 15 & 20 & 28 \\
\hline$\rho\left[\mathrm{kg} / \mathrm{m}^{3}\right]$ & 14.43 & 39.85 & 78.25 & 39.66 & 78.22 & 78.61 & 69.09 \\
\hline $\mathrm{T}\left[{ }^{\circ} \mathrm{K}\right]$ & 301.3 & 300.7 & 301.0 & 301.7 & 299.9 & 301.0 & 306.1 \\
\hline Mach [-] & 0.075 & 0.054 & 0.029 & 0.082 & 0.044 & 0.058 & 0.08 \\
\hline $\mathrm{Ti}[\%]$ & $\begin{array}{l}\mathrm{Ti} 1=0.5129 \\
\mathrm{Ti} 2=0.3200 \\
\mathrm{Ti} 3=0.0864\end{array}$ & $\begin{aligned} \mathrm{Ti} 1 & =0.5451 \\
\mathrm{Ti} 2 & =0.4250 \\
\mathrm{Ti} 3 & =0.1614\end{aligned}$ & $\begin{aligned} \mathrm{Ti} 1 & =0.8058 \\
\mathrm{Ti} 2 & =0.4600 \\
\mathrm{Ti} 3 & =0.1988\end{aligned}$ & $\begin{aligned} \mathrm{Ti} 1 & =0.9057 \\
\mathrm{Ti} 2 & =0.4100 \\
\mathrm{Ti} 3 & =0.1988\end{aligned}$ & $\begin{array}{l}\mathrm{Ti} 1=1.1877 \\
\mathrm{Ti} 2=0.4500 \\
\mathrm{Ti} 3=0.2448\end{array}$ & $\begin{array}{l}\mathrm{Ti} 1=2.2790 \\
\mathrm{Ti} 2=0.5100 \\
\mathrm{Ti} 3=0.3015\end{array}$ & $\begin{aligned} \mathrm{Ti} 1 & =2.3944 \\
\mathrm{Ti} 2 & =0.5500 \\
\mathrm{Ti} 3 & =0.3346\end{aligned}$ \\
\hline
\end{tabular}

Table 1. Cases used in the blind test campaign 


\section{Description of the Participated Codes}

The participating codes are categorized in two groups as full CFD and panel codes. Not all the participants sent contribution to all cases. The names of the codes and the participants and the cases computed are summarized in Table 1. In the following sections, short explanations are given for each code and the specific settings used to produce the results of this blind test campaign.

\begin{tabular}{|c|c|c|c|c|c|c|c|c|c|}
\hline & \multirow[b]{2}{*}{$\mathrm{P}_{\mathrm{t}}[$ bars $]$} & $\begin{array}{l}\text { Test } 1 . \\
\operatorname{Re}=3 \mathrm{mil}\end{array}$ & $\begin{array}{c}\text { Test } 2 . \\
\operatorname{Re}=6 \text { mil- } 1\end{array}$ & $\begin{array}{c}\text { Test } 3 . \\
\text { Re=6mil-2 }\end{array}$ & $\begin{array}{c}\text { Test } 4 . \\
\operatorname{Re}=9 \text { mil- } 1\end{array}$ & $\begin{array}{c}\text { Test } 5 \text {. } \\
\operatorname{Re}=9 \text { mil- } 2\end{array}$ & $\begin{array}{l}\text { Test } 6 . \\
\operatorname{Re}=12 \mathrm{mil}\end{array}$ & $\begin{array}{c}\text { Test } 7 \\
\mathrm{Re}=15 \mathrm{mil}\end{array}$ \\
\hline & & & 12 & 34 & 67 & 34 & 67 & 67 & 60 \\
\hline & & $\mathrm{V}_{\text {tunnel }}[\mathrm{m} / \mathrm{s}]$ & 25.6 & 19 & 10 & 28.6 & 15 & 20 & 28.4 \\
\hline \multirow{3}{*}{\multicolumn{2}{|c|}{ DTU/EllipSys }} & $\begin{array}{l}\text { Fully } \\
\text { turbulent }\end{array}$ & & & & & & & \\
\hline & & Transition & Yes & Yes & Yes & Yes & Yes & Yes & Yes \\
\hline & & $\begin{array}{l}\text { Fully } \\
\text { turbulent }\end{array}$ & & & & & & & \\
\hline \multirow{5}{*}{ 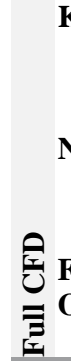 } & & Transition & Yes & Yes & Yes & Yes & Yes & Yes & Yes \\
\hline & NTUA/Mapflow & $\begin{array}{l}\text { Fully } \\
\text { turbulent }\end{array}$ & & & & & & & \\
\hline & & Transition & Yes & Yes & Yes & Yes & Yes & Yes & Yes \\
\hline & Forwind-IWES/ & $\begin{array}{l}\text { Fully } \\
\text { turbulent }\end{array}$ & Yes & Yes & Yes & Yes & Yes & Yes & Yes \\
\hline & OpenFOAM & Transition & & & & & & & \\
\hline \multirow{5}{*}{ 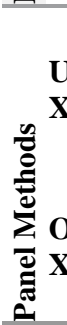 } & USTUTT/ & $\begin{array}{l}\text { Fully } \\
\text { turbulent }\end{array}$ & & & & & & & \\
\hline & XFOILvUSTUTT & & & & & & & & \\
\hline & & Transition & Yes & Yes & Yes & Yes & Yes & Yes & Yes \\
\hline & RE Catapult/ & $\begin{array}{l}\text { Fully } \\
\text { turbulent }\end{array}$ & & & & & & & \\
\hline & & Transition & Yes & Yes & Yes & Yes & Yes & Yes & Yes \\
\hline
\end{tabular}

Table 2. Participating codes to the blind test campaign

\section{A. Full CFD codes}

Four full CFD codes from four participants are introduced below.

\section{EllipSys2D Code from DTU (Technical Univ. of Denmark)}

EllipSys2D is an in-house incompressible finite volume Reynolds-Averaged Navier-Stokes (RANS) flow solver developed at DTU, see [1],[2],[3]. The flow variables are collocated in the mesh to facilitate complex mesh geometries. The SIMPLE algorithm is used to, see [4], and the pressure correction equation is solved using the RhieChow algorithm to avoid odd/even pressure decoupling, see [5] and is accelerated by a multi-grid technique. The convective terms are discretized using the QUICK scheme, as given by [6]. The code is parallelized using a multiblock structure grid, where the problem can be distributed across multiple processors. The communication of data between each processor is done through the MPI libraries.

The present 2D computations are all computed on an O-mesh of 512 times 256 in chord wise and normal direction. The high grid density is used to assure grid independent results on the coarser level-2 grid of 256 times 128 cells. The computations are performed in steady state and Menter's k-omega SST model is used to describe the boundary layer turbulence [7]. The laminar to turbulent transition process is handled by the semi-empirical $\mathrm{e}^{\mathrm{N}}$ method by Drela-Giles [8], as implemented by Michelsen [9]. A full database implementation is also available, but is not applied in the present study. 


\section{TAU Code from University of Kiel}

The DLR TAU-Code [11] is a software system for the prediction of compressible, three dimensional, viscous or inviscid flows. The flow regime goes from subsonic to hypersonic. A high number of different turbulence models are supplied by TAU. Using hybrid unstructured meshes the prediction of flows about complex geometry is possible. TAU is composed of different modules for pre-processing, solving, mesh adaptation and deformation, transition and more. For mesh generation and post-processing external tools are needed. The use of TAU on parallel computers is based on the message passing interface (MPI). Originally, TAU was developed for numerical simulations of aircraft-type configurations but can be applied to a wide range of problems.

For these calculations, the release TAU 2014.2.0 and the TAU transition module V9.30 (2014.2.0) were used. Solver setup for computations are listed below:

- Solver Compressible RANS

- Turbulence model Menter SST k-omega two-equation model from 2003

- Transition prediction TS transition predicted by $\mathrm{e}^{\mathrm{N}}$-Method with $\mathrm{N}$-factors from a linear stability solver

- Low Ma Preconditioning PrimNew with Matrix dissipation for central flux

The mesh was generated using ICEM CFD with non-dimensional distance from the first node equal to 3.10-7 corresponding to y+ equal to 0.2. 257 nodes are used to model the airfoil surface and far field is located at 22 chord lengths. The total number of grid points was 49120 . The far field boundary condition was modeled with reference settings according to Table 1 from AVATAR blind test documentation, respectively. The airfoil was modeled as a viscous wall. The boundary layers were assumed to be partially laminar and partially turbulent, with a laminarturbulent transition on either side. The position of the transition was predicted using the $\mathrm{e}^{\mathrm{N}}$ method with $\mathrm{N}$-factors calculated by a linear stability solver.

The critical $\mathrm{N}$ factor was calculated from turbulence intensity by using Mack's correlation $\mathrm{N}=-8.43-2.4 \mathrm{TI}$

Description of numerical solution algorithm is also listed here:

- Relaxation solver Backward-Euler (implicit)

- Preconditioning PrimNew

- Central flux dissipation scheme Matrix dissipation

- Multigrid cycle 5v

For further details on the solution algorithm in TAU please refer to [9] and [11].

\section{Mapflow code from NTUA (National Technical Univ. of Athens)}

MaPFlow is an unsteady Reynolds-Averaged Navier Stokes (URANS) solver developed at the NTUA's Aerodynamics Laboratory. It is a multiblock, compressible, cell centered CFD Solver that can use both structured and unstructured grids. The convective fluxes are discretized using the approximate Riemann solver of Roe [12] with Venkatakrishnan limiter [13], while the viscous fluxes are discretized using a central $2^{\text {nd }}$ order scheme.

Turbulence closures implemented on MaPFlow include the one equation turbulence model of Spalart (SA) [14] as well as the two equation turbulence model of Menter [15](k-omega SST).

MaPFlow can handle both steady and unsteady flows. Time integration is achieved in an implicit manner permitting large CFL numbers. The unsteady calculations use a $2^{\text {nd }}$ order time accurate scheme combined with the dual time-stepping technique [19] to facilitate convergence when complex unsteady flows with moving /deforming geometries are considered. Additionally, flows in the incompressible region are feasible using Low Mach Preconditioning [20]. MaPFlow is parallelized using the MPI library in a multi-block fashion in which each processor solves a partition of the original computational domain.

The transition model used for this campaign is the Granville/Schlichting transition prediction, the method is fully described in [17]. It is based on boundary layer characteristics and utilizes the Polhausen variables to compute them using the velocity on the edge of the boundary layer as input, the latter being computed using the pressure coefficient value on the viscous wall derived by the RANS solver. The instability and transition points are then defined using empirically calibrated diagrams proposed by Granville. The simulations used the SST k-omega model of F.Menter.

The grid file created for the simulation is an o-type grid and has 505 elements around the airfoil and 197 elements in the normal direction. The first cell was always less than $1 \mathrm{e}-05$ resulting in a $\mathrm{Y}+<1$ at all times. 


\section{OpenFOAM code from ForWind/IWES}

IWES and ForWind used the open source CFD library OpenFOAM (version 2.3.1 ${ }^{1}$ ) for their simulations. The SIMPLE algorithm [4] is used to solve the steady incompressible finite volume RANS equations. The pressure equation is solved by means of a "quasi" Rhie-Chow [25] algorithm to suppress oscillation and accelerated via a generalized-algebraic-multi-grid technique. The convective terms are discretized using a second order bounded scheme. The execution is parallelized using the OpenMPI library ${ }^{2}$. Simulations were performed using the Menter's k-omega SST turbulence model [15]. The two-dimensional fully structured O-shape numerical domain featured 800 x $150=120.000$ cells with an outer radius of 50 chord lengths, keeping the value of the Y+ below 1 .

These simulations are provided for comparison against the experimental data set obtained by tripping the boundary layer near the leading edge. They must be regarded as a reference for fully turbulent cases, which are representative for the increased surface roughness due to the accumulation of dust and dirt particles on the blade's leading edge. Although these numerical results do not aim to be compared against the experimental data sets obtained with natural transition, they are included in the comparisons anyways to be used as reference for fully turbulent simulations.

\section{B. Panel Codes}

\section{XFOILvUSTUTT from USTUTT}

For the present blind test analyses an extended version of the airfoil design and analysis code XFOIL was applied by University of Stuttgart (USTUTT or UoS). XFOIL is a well established and widely used tool being developed by Drela [8]. The method is based on a coupled panel boundary-layer procedure which offers inverse, mixed-inverse design or direct analysis capabilities for single-element airfoils. The peculiarity of this method is that the governing Laplace equation for the outer-flow computation is directly coupled with the integral boundary-layer equations taking the boundary-layer displacement-effect into account. The coupled system of equations is solved simultaneously by means of an iterative Newton method.

The implemented first order integral boundary-layer method is based on a numerical integration of the integral momentum and energy equation. For turbulent flows an additional lag-equation is solved which accounts for nonequilibrium effects. The closure for turbulent flows is based on an evaluation of the Swafford boundary-layer profile family. For transition prediction a simplified $\mathrm{e}^{\mathrm{N}}$ envelope method is used in the standard XFOIL code. In the version used for the present studies a full $\mathrm{e}^{\mathrm{N}}$ method that determines the frequency-dependent TS amplification was linked. The code enables the simulation of laminar separation bubbles and to account for separation without reattachment. Due to the linearized approach to simulate the boundary-layer displacement effect and the fundamental problems associated with the (time-averaged) simulation of separated flow, the reliability of the XFOIL prediction in the stall and post-stall regime is limited and requires a careful, experience-based interpretation.

\section{XFOIL from ORE Catapult}

XFOIL version 6.96 used for the analysis.

\section{Results}

The plots are presented separately for the full CFD codes and for panel codes. For the panel code results, a full CFD code is also put in the graphs in order to give the reader the impression of how they perform compared to the full CFD codes.

\section{A. Lift Predictions}

In Figure 4, $c_{1}$ predictions of full CFD codes are compared with the experiments for $\operatorname{Re}=9 \times 10^{6}$ and $\operatorname{Re}=15 \times 10^{6}$. The predictions from TAU code are only available for the linear lift region. In this region, both for the positive and the negative angles of attacks, there is a good agreement between the predictions and the experiments. Although the predictions also agree with each other about the maximum and minimum lift angle and more or less about the maximum lift value, they are not able to predict the maximum lift value and not able to capture the drop on the lift observed during the experiments. This can be explained by the fact that stall is an unsteady 3D phenomenon which is influenced heavily from the model aspect ratio and the wind tunnel and the computations were only performed for a 2D steady state condition without taking into account these aspects. Nevertheless, EllipSys and OpenFOAM predictions seem to come closer to the experiments again for the post stall region. Another observation in these

1 http://openfoam.org/version/2-3-1/

2 https://www.open-mpi.org/ 
figures is that the mismatch between the predictions and the experiments is slightly less in the case of $\operatorname{Re}=15 \times 10^{6}$. This fits with the expectations as the flow is more turbulent in the case of $\operatorname{Re}=15 \times 10^{6}$ and therefore, the effect of the laminar-turbulent transition is less dominant than in the case of lower Re numbers.

Figure 5 shows the lift predictions of XFOIL codes compared for the same conditions and a full CFD code. For the linear lift region, XFOIL codes also agree well with the experiment and with the full CFD predictions. Although they are not able to capture the drop in the lift, XFOIL code from USTUTT seem to be able to predict the maximum lift and the angle of it better than the full CFD codes. This indicates that the full $\mathrm{e}^{\mathrm{N}}$ method is probably working better than the simplified version as implemented in the standart XFOIL and in EllipSys, to capture the maximum lift and the stall angle. XFOIL predictions also agree better with the experiments in the case of higher Re number similarly as in the full CFD codes.

Looking at the trends in the $c_{\operatorname{lmax}}$ and the angle of attack with changing Re numbers in Figure 6 , it can be noted that although the value of the $c_{\operatorname{lmax}}$ is not captured well, its trend with increasing Re numbers is well predicted. The lift curve slope does not change with increasing Re numbers which matches with the predictions from all codes.
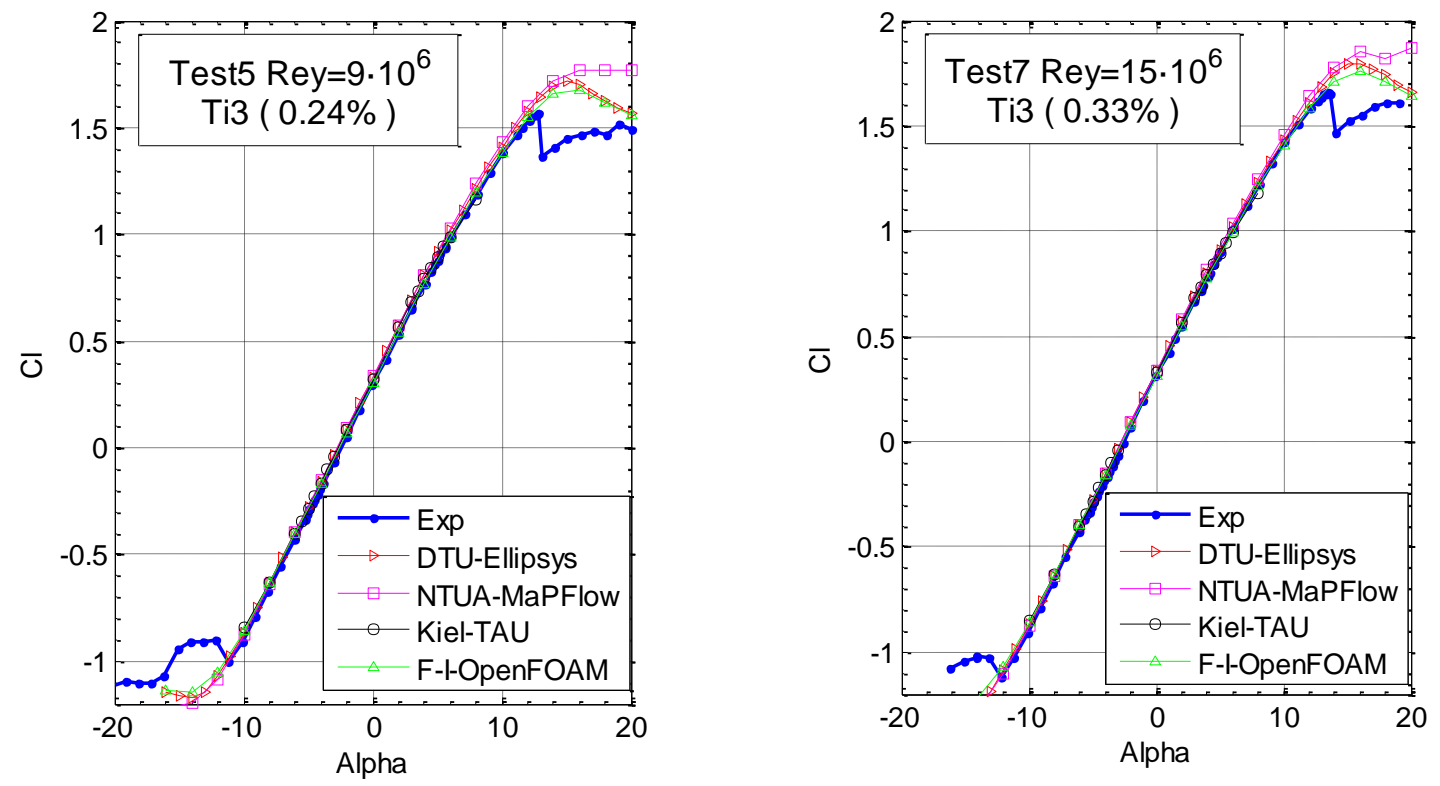

Figure 4. $c_{1}$ vs Alpha (angle of attack) comparisons of full CFD codes for 9 and 15 million Re numbers
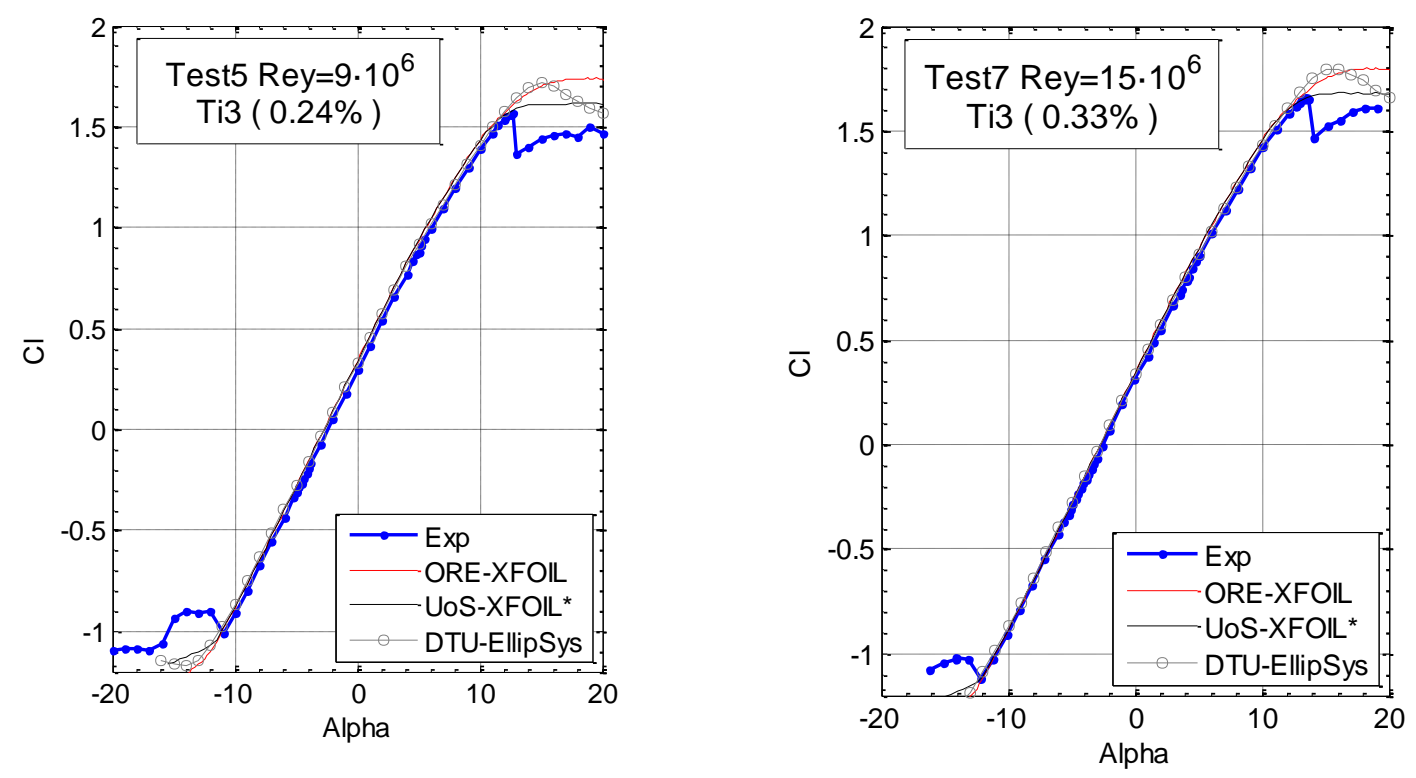

Figure 5. $c_{1}$ vs Alpha comparisons of Panel codes and one full CFD code for 9 and 15 million Re numbers 

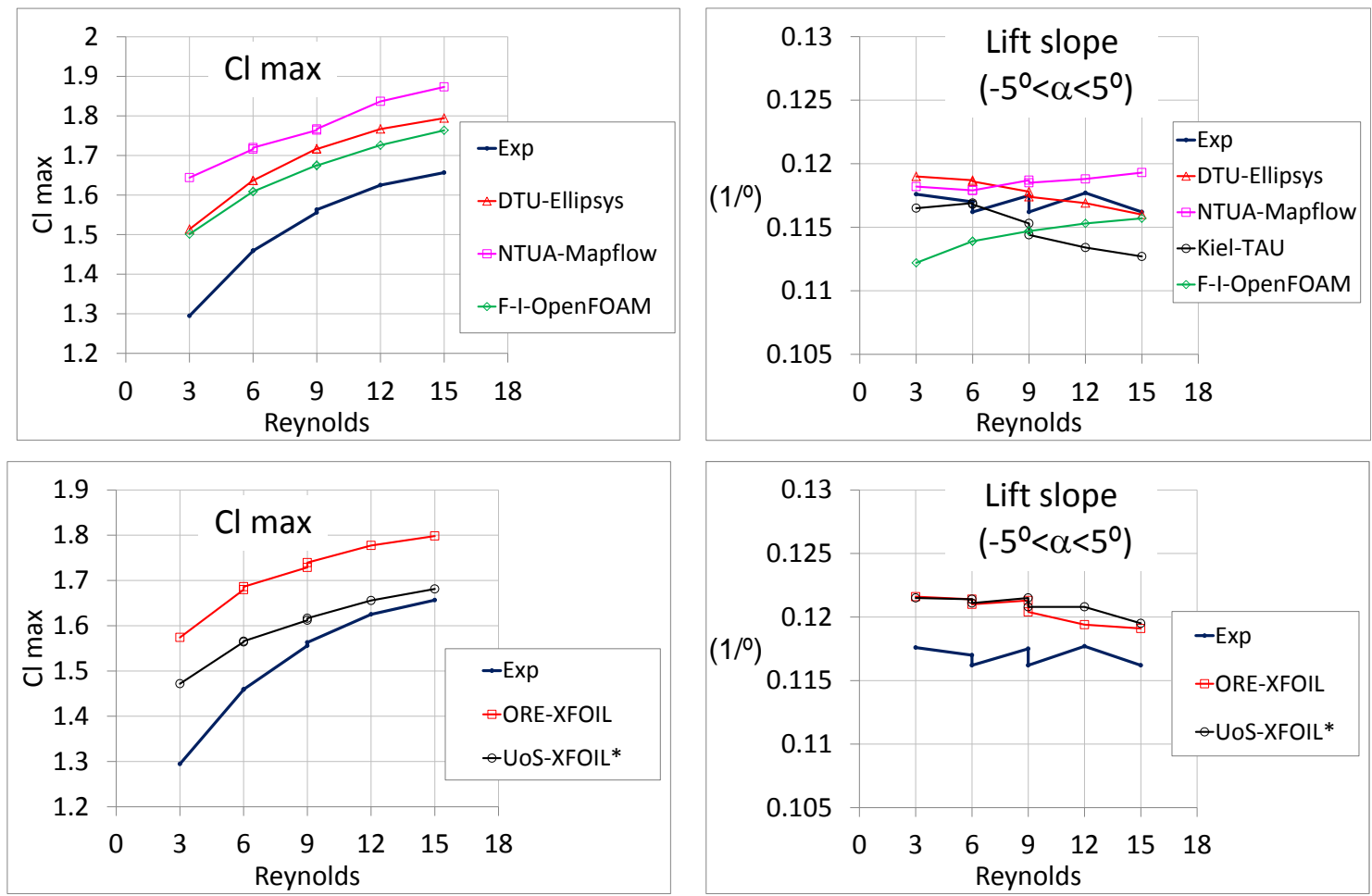

Figure 6. Comparison of $c_{\operatorname{lmax}}$ and lift slope Reynolds number trends of the codes.

\section{B. Drag Predictions}

The drag predictions for $\operatorname{Re}=9 \times 10^{6}$ and $\operatorname{Re}=15 \times 10^{6}$ are shown in Figure 7 in terms of $c_{1}$ vs $c_{d}$ drag counts. The laminar drag bucket is visible both at the $\operatorname{Re}=9 \times 10^{6}$ and $\mathrm{Re}=15 \times 10^{6}$ cases, therefore the turbulence of the incoming flow plays an important role for the drag predictions especially in this region.

TAU, MapFlow and EllipSys predictions show a clear laminar drag bucket for both for $\operatorname{Re}=9 \times 10^{6}$ and $\operatorname{Re}=15 \times 10^{6}$ which is very close to the experiment. Moreover, the actual drag values seems to be predicted reasonably well. OpenFOAM analysis are done for the fully turbulent case therefore it is expected that these results will show much higher drag prediction. On the other hand, the decrease in the drag going from $\operatorname{Re}=9 \times 10^{6}$ to $\operatorname{Re}=15 \times 10^{6}$ is well predicted by OpenFOAM. The predictions of the sharp lower corner of the laminar drag bucket can be further improved by using more angle of attack values and slightly different $\mathrm{N}$ values. Outside the laminar drag bucket, EllipSys results show a consistent underestimation both for the negative and positive angles of attack, TAU overestimates the drag in the upper corner slightly. MaPFlow shows slight overestimation of drag both for the entire laminar drag bucket where outside this region there is overestimation only for the positive angles of attack. Although these predictions can further be improved by using a more sutable description of the inflow turbulence and possibly with a mesh refinement study, the agreement between the analysis and the tests is improved for the $\mathrm{Re}=15 \times 10^{6}$ case. This is expected as the effect of transition models should be less in higher Reynolds numbers. However, this might also indicate that especially Granville/Schlichting transition prediction is probably working better for the higher Reynolds number range.

Similarly, XFOIL results show a good agreement with the experiments except for a consistent underprediction of the drag along the entire drag curve as shown in Figure 8. Especially the shape of the laminar drag bucket is captured very well by the both XFOIL versions. These comparisons show that although the drag is not accurately predicted, XFOIL codes are still useful to obtain the right trends in this high Reynolds number ranges. This is a very good news because these codes are commonly used in the wind industry and they will remain to be useful as a quick decision making tool to estimate the right trends in designing or analyzing the airfoils of the very large wind turbine blades. 

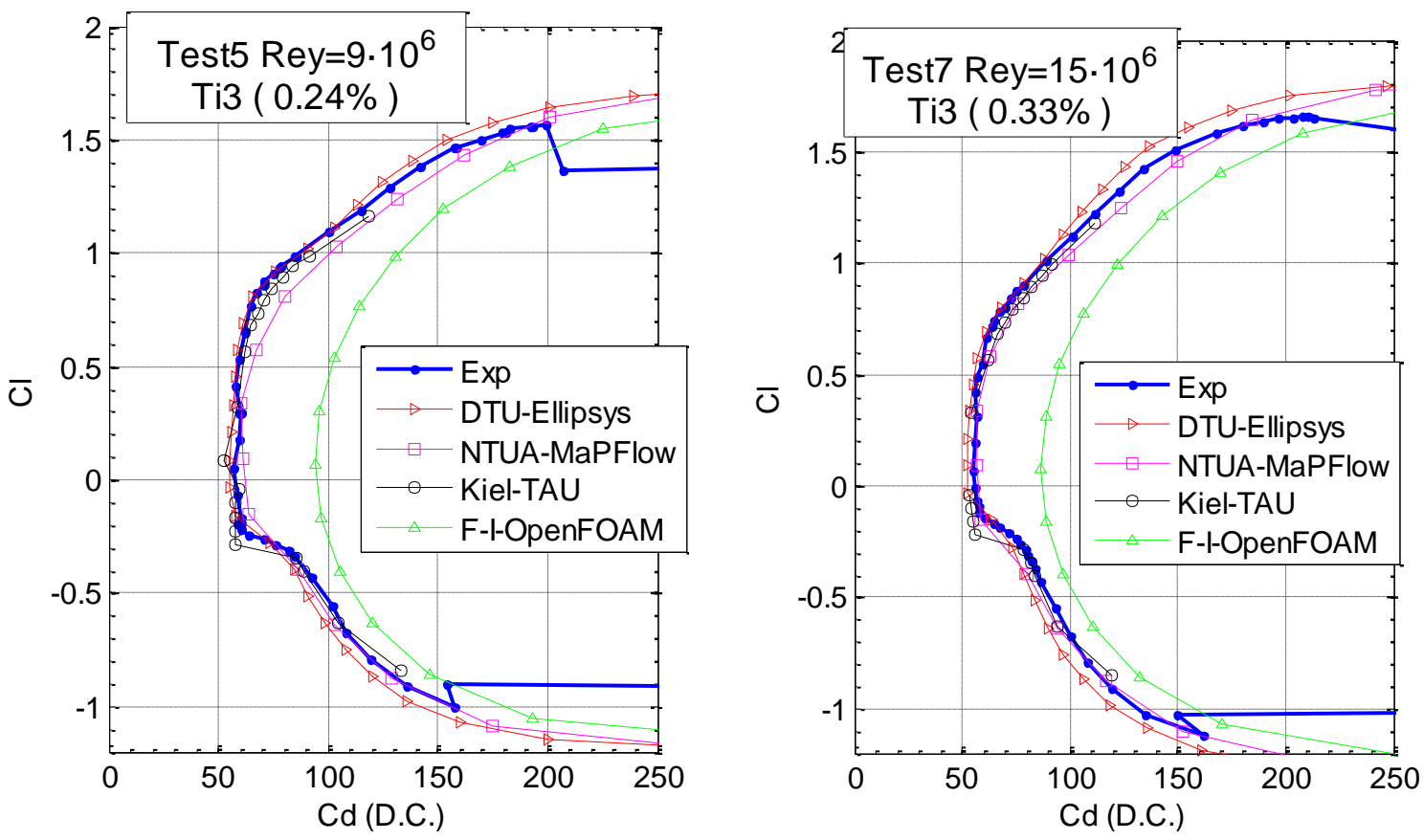

Figure 7. $c_{1}$ vs $c_{d}$ comparison of full CFD codes at 9 and 15 million Re number
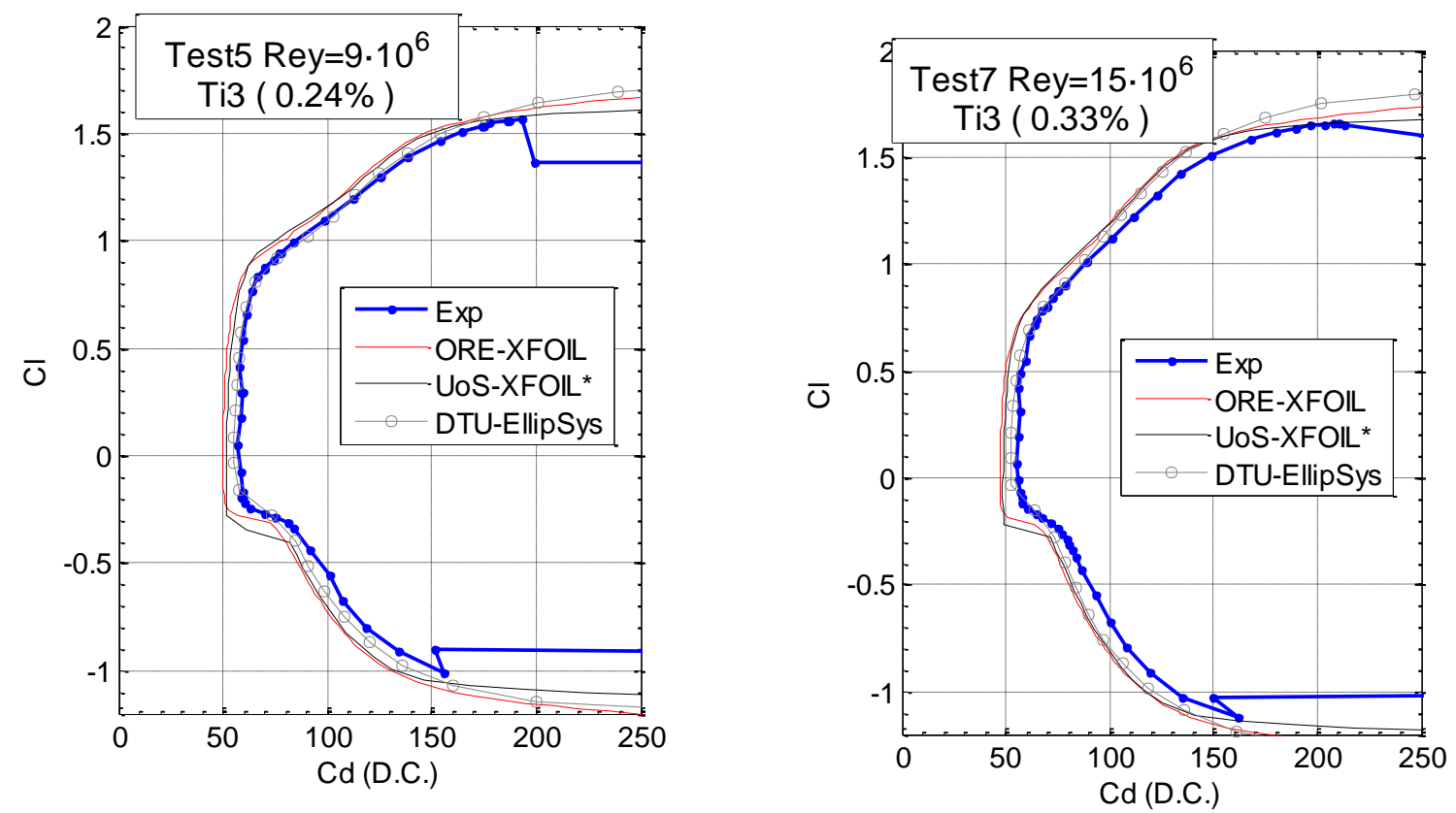

Figure 8. $c_{1}$ vs $c_{d}$ comparison of panel codes and a full CFD code at 9 and 15 million Re number

\section{Glide Ratio $\left(\mathbf{c}_{\mathbf{l}} / \mathbf{c}_{\mathrm{d}}\right)$ Predictions}

Glide ratio is an important parameter used in the airfoil and blade designs. Glide ratio versus positive angles of attack is plotted in Figure 9 for the full CFD codes and in Figure 10 for the XFOIL codes. TAU predictions are almost on top of the experiment except a slight but persistent underestimation after around 2-3 degrees for both Re numbers. MaPFlow results are similar except that the underestimation is higher and the trend is not well captured although, this changes for $\mathrm{Re}=15 \times 10^{6}$ where the trend is well captured and the underestimation is less. Ellipsys results are very close to the experiment with slight overprediction of the $c_{1} / c_{d}$ until $c_{1} / c_{d}$ max which is well predicted. For higher angles of attack, $c_{1} / c_{d}$ is overpredicted, and this overprediction is higher in $\operatorname{Re}=15 \times 10^{6}$. The test results show that the peak at the glide ratio curve disappears going from $\operatorname{Re}=9 \times 10^{6}$ to $\mathrm{Re}=15 \times 10^{6}$ and the glide ratio curve flattens for a range of the angle of attacks. This phenomenon is well captured with all three codes. Fully turbulent 
OpenFOAM results able to predict an increase in $c_{1} / c_{d}$ going from $\operatorname{Re}=9 \times 10^{6}$ to $\operatorname{Re}=15 \times 10^{6}$ which is in line with the expectations and confirmed with the test results for the region around 10 degrees where there transition models are less significant. Both XFOIL codes predict the $c_{1} / c_{d}$ trend for both Re numbers quite well. Despite the consistent overestimation of the actual values by the both codes, USTUTT XFOIL predictions are somewhat closer to the experiments. Especially for the high Re number case, it is remarkable that the $c_{l} / c_{d}$ trend is also captured as both XFOIL codes are able to predict the angle of the glide ratio peak for both Re numbers. These results support the argument above about the usage of the XFOIL codes for the design and analysis of the airfoils for very large wind turbine blades as a quick decision making tool.
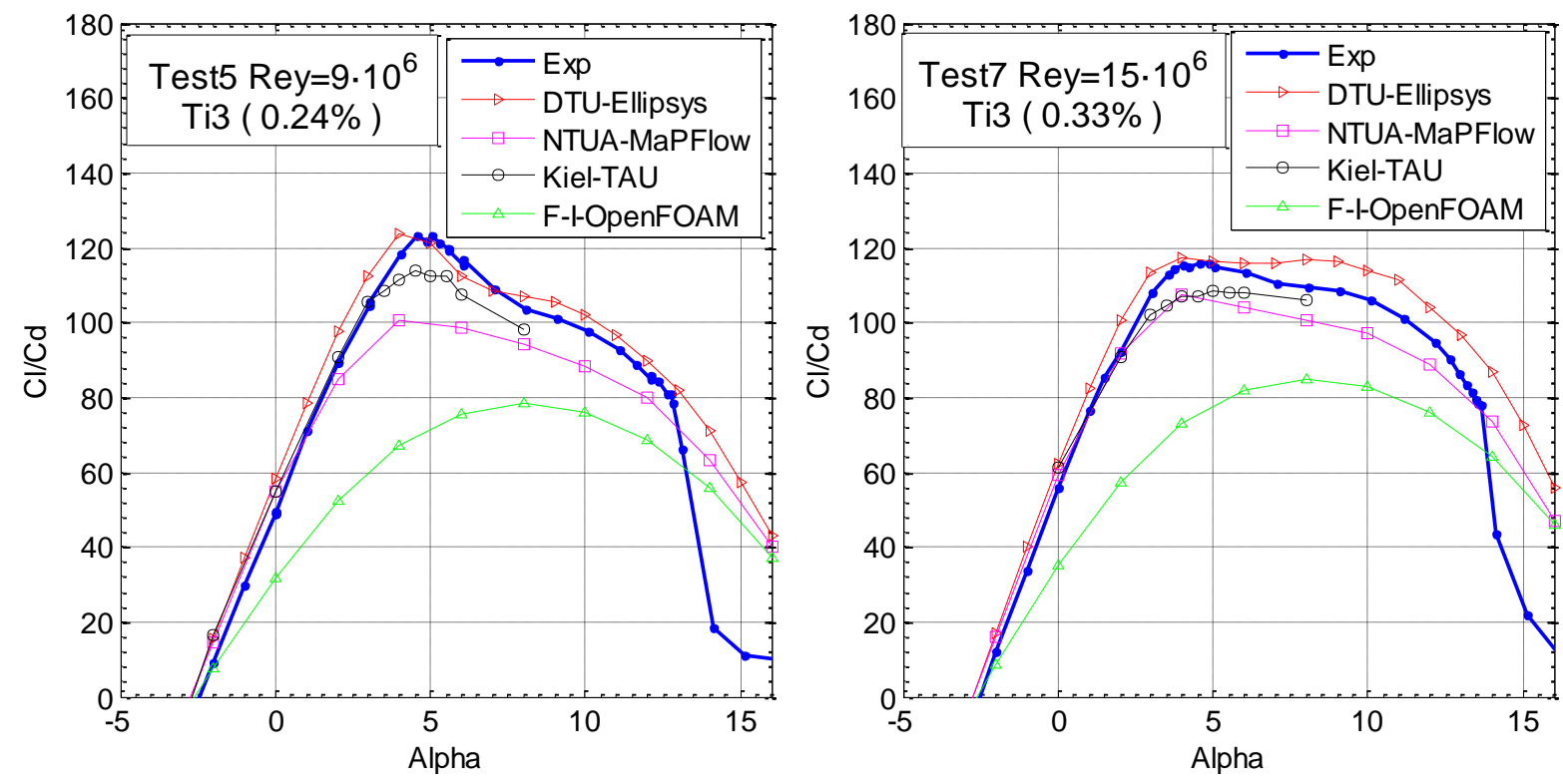

Figure 9. Glide ratio comparison of full CFD codes at 9 and 15 million Re numbers
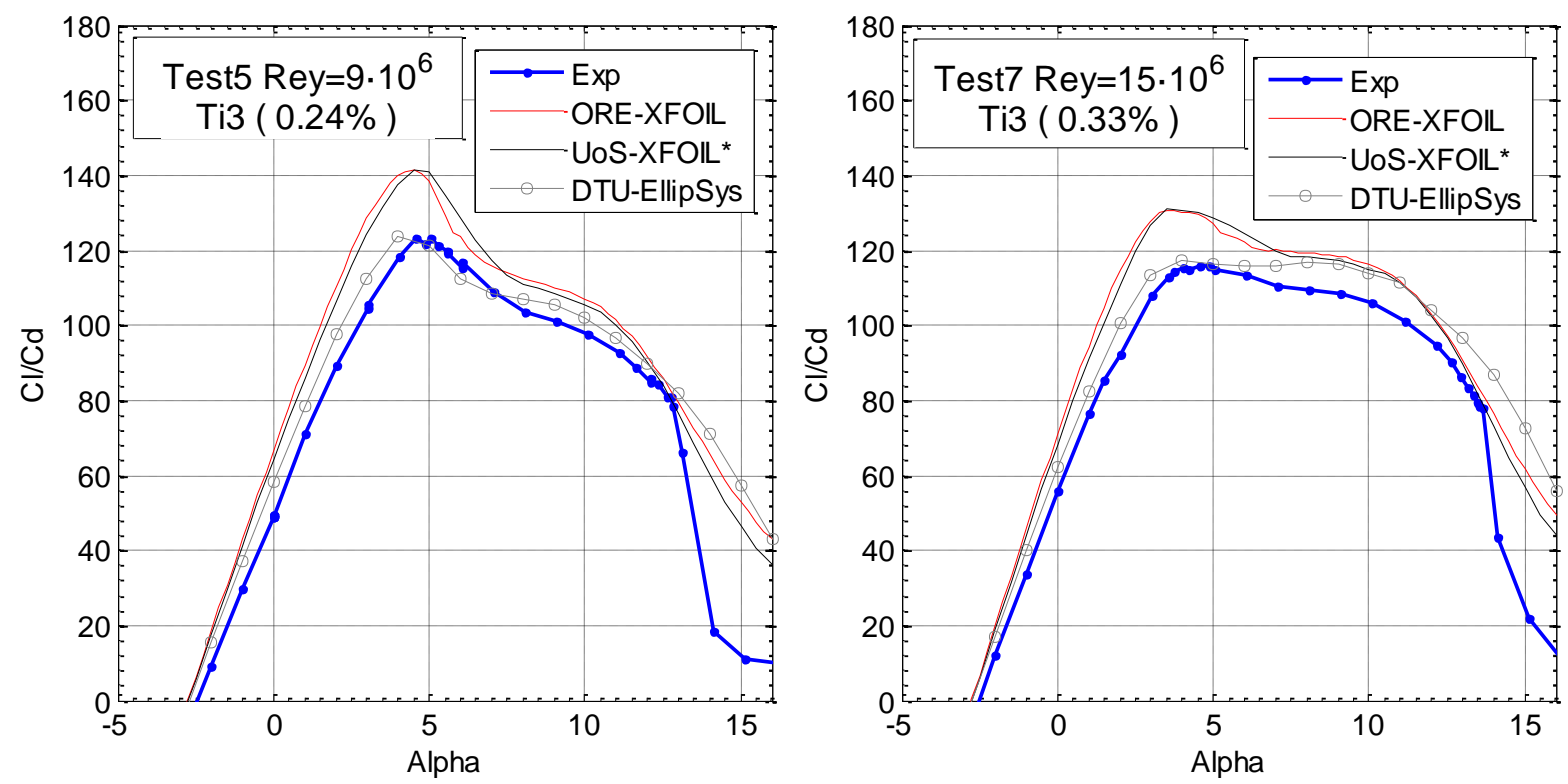

Figure 10. Glide ratio comparison of the panel codes and one full CFD code at 9 and 15 million Re numbers 


\section{Effect of Inflow Turbulence}

The effect of different inflow turbulence in the prediction accuracy is shown in Figure 11 using the computational results of the glide ratio for three different inflow turbulence intensity values both for the $\operatorname{Re}=3 \times 10^{6}$ case using EllipSys results and for $\mathrm{Re}=12 \times 10^{6}$ case using TAU results. It can be observed that the glide ratio predictions are strongly dependent on the choice of the inflow turbulence. Moreover, this dependency is stronger for the higher Re numbers. Using a different inflow turbulence value does not only change the prediction accuracy of the actual $c_{l} / c_{d}$ value but also the glide ratio trend changes significantly for the high Re number case. This can play an important role in the airfoil designs for large wind turbines where the design and the operating Re numbers are much higher than $3 \times 10^{6}$. If the correct inflow conditions of the airfoil is not used during the airfoil design, the airfoil will have a different shape and a different $c_{1} / c_{d} \max$ value and angle then it is actually designed for. Therefore, especially for the large wind turbine airfoil designs, the choice of the correct inflow conditions can be crucial. This results suggest that there is an urgent need for more detailed investigation about the inflow conditions of the large wind turbine airfoils and the accuracy of the prediction tools for these inflow conditions.
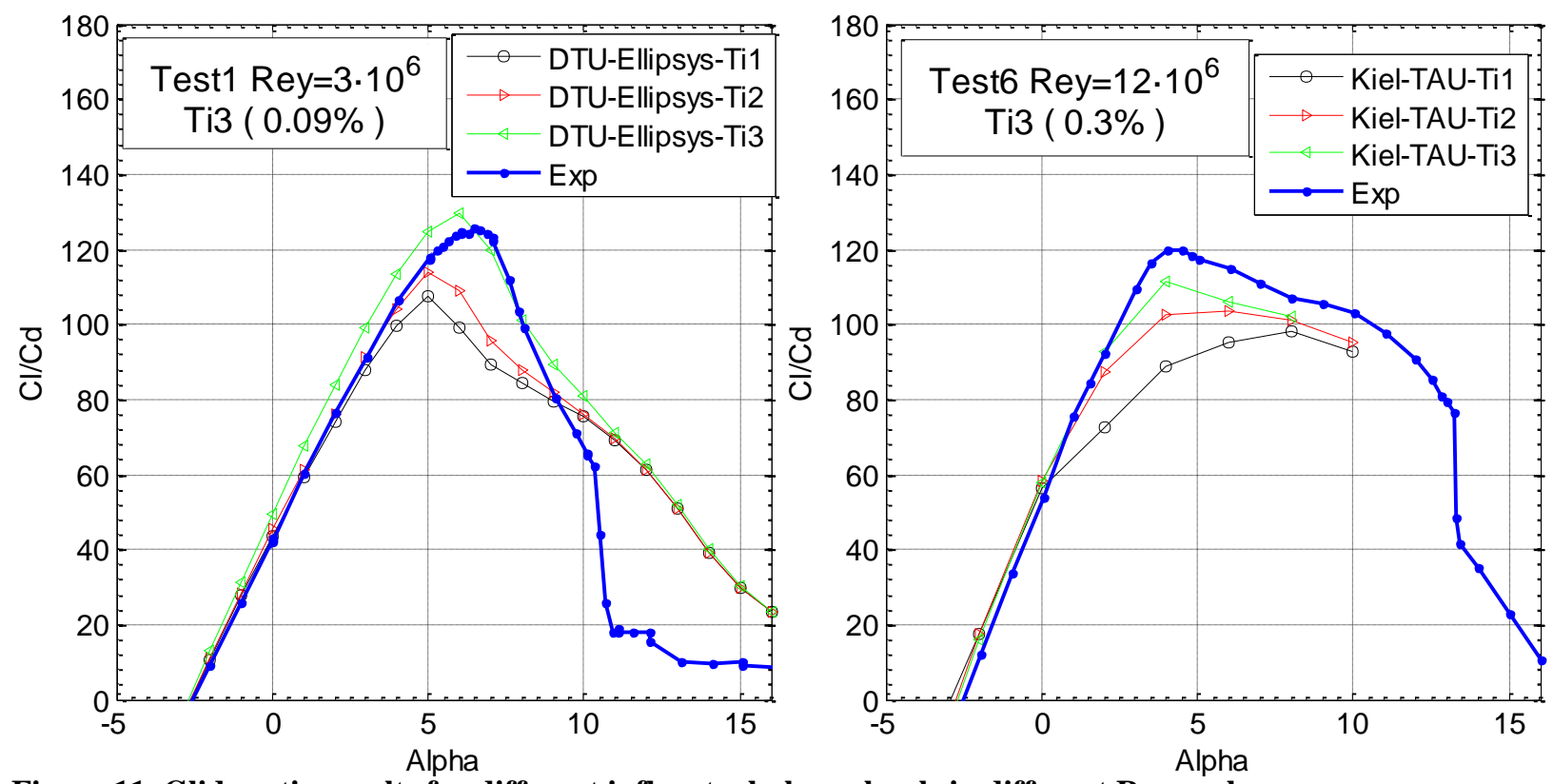

Figure 11. Glide ratio results for different inflow turbulence levels in different Re numbers.

\section{Conclusions}

The results of the blind test campaign for the selected cases of the high Reynolds number tests done in DNWHDG wind tunnel was presented in this paper. The main conclusions are summarized here.

- All codes are able to predict the linear lift region quite well however, the maximum lift and the maximum lift angle are always overpredicted.

- Low drag region is predicted well with $\mathrm{e}^{\mathrm{N}}$ transition model in general, except the corners. Better agreement can be otained by using more accurate inflow turbulence and choosing more angle of attack values to capture the corners.

- The drag values outside the laminar drag bucket is over predicted mostly except EllipSys and XFOIL codes where in this case they are underpredicted.

- Glide ratio is well predicted with EllipSys and TAU, but not with the other codes. Panel codes can reproduce the general behaviour but always overpredict the absolute values.

- Reynolds number effects in $\mathrm{c}_{\mathrm{l}} / \mathrm{c}_{\mathrm{d}} \max , \mathrm{c}_{\mathrm{dmin}}$ and $\mathrm{c}_{\operatorname{lmax}}$ are well predicted with $\mathrm{e}^{\mathrm{N}}$ transition model, even if the absolute values are different.

- MaPFlow underpredicts the glide ratio with a maximum value that increases with Re which is contrary to the measurements. Part of this is attributed to the transition modelling.

- Fully turbulent OpenFOAM analysis predict the lift and the general trends with increasing Re number well except $\mathrm{c}_{\mathrm{l}} / \mathrm{c}_{\mathrm{d}}$ trend.

- $\mathrm{e}^{\mathrm{N}}$ transtion model is able to predict Re effects quite well.

11

American Institute of Aeronautics and Astronautics 
- XFOIL codes are able to predict the general trends very well where the $c_{\operatorname{lmax}}$ values are at the same level as CFD predictions. Despite their worse accuracy mainly in the drag values, they can still be used as a quick decision making tool in the airfoil design or analysis.

The sensitivity of the glide ratio to the inflow turbulence calls for deeper investigation as the glide ratio is the most important parameter during the airfoils designs in wind turbine applications. In order to have more reliable and efficient wind turbines for $10 \mathrm{MW}+$ range, additional research about the correct inflow conditions in airfoil design is crucial.

\section{Acknowledgments}

This work is partially funded by European Union 7th framework program AVATAR Project with the agreement number EU FP7-Energy-2013-1 / n 608396. NTUA computations were supported by computational time granted from the Greek Research \& Technology Network (GRNET) in the National HPC facility - ARIS - under project "MAPWIND" with ID pr002028.

\section{References}

[1] J.A. Michelsen. Basis3D - a Platform for Development of Multiblock PDE Solvers. Technical Report AFM 92-05, Technical University of Denmark, Department of Fluid Mechanics, Technical University of Denmark, December 1992.

[2] J.A. Michelsen. Block structured Multigrid solution of 2D and 3D elliptic PDE's. Technical Report AFM 94-06, Technical University of Denmark, Department of Fluid Mechanics, Technical University of Denmark, May 1994.

[3] N.N. Sørensen. General Purpose Flow Solver Applied to Flow over Hills. Ris $\varnothing-R-827-E N)$, Ris $\varnothing$ National Laboratory, Roskilde, Denmark, June 1995.

[4] S.V. Patankar and D.B. Spalding. A Calculation Prodedure for Heat, Mass and Momentum Transfer in ThreeDimensional Parabolic Flows. Int. J. Heat Mass Transfer, 15:1787, 1972.

[5] C.M. Rhie. A numerical study of the flow past an isolated airfoil with separation. PhD thesis, Univ. of Illinois, UrbanaChampaign, 1981

[6] B.P. Leonard. A stable and accurate convective modelling procedure based on quadratic upstream interpolation. Comput. Meths. Appl. Mech. Eng., 19:59--98, 1979.

[7] F.R. Menter. Zonal Two Equation k-omega Turbulence Models for Aerodynamic Flows. AIAA paper 1993-2906, 1993.

[8] M. Drela and M.B. Giles. Viscous-Inviscid Analysis of Transonic and Low Reynolds Number Airfoils. AIAA Journal, 25(10):1347--1355, October 1987.

[9] TAU-Code User Guide, Manual for Release 2014.2.0, Deutsches Zentrum für Luft- und Raumfahrt e. V., 2014

[10] Transition module (V9.30) User guide (V1.04), Manual, Deutsches Zentrum für Luftund Raumfahrt e. V., 2009

[11] Technical Doc/ of the DLR TAU-Code; Technical report, Institute of Aerodynamics and Flow Technology, DLR

[12] Roe, P., Characteristic-based schemes for the Euler equations. Annual review of fluid mechanics, 1986. 18(1): p. 337 365.

[13] V, V., On the accuracy of limiters and convergence to steady state solutions, in 31st Aerospace Sciences Meeting. 1993, American Institute of Aeronautics and Astronautics.

[14] Spalart, P.R. and S.R. Allmaras, A one equation turbulence model for aerodinamic flows. AIAA journal, 1992.94.

[15] Menter, F.R., Two-equation eddy-viscosity turbulence models for engineering applications. AIAA journal, 1994. 32(8): p. $1598-1605$.

[16] Langtry, R.B. and F.R. Menter, Correlation-based transition modeling for unstructured parallelized computational fluid dynamics codes. AIAA journal, 2009. 47(12): p. 2894-2906.

[17] Schlichting, H., Boundary-layer theory. 1968.

[18] Van Ingen, J., A suggested semi-empirical method for the calculation of the boundary layer transition region. 1956, Delft University of Technology.

[19] Vatsa, V.N., M.H. Carpenter, and D.P. Lockard, Re-evaluation of an optimized second order backward difference (BDF2OPT) scheme for unsteady flow applications. AIAA Paper, 2010. 122: p. 2010.

[20] Eriksson, L.-E. A preconditioned Navier-Stokes solver for low Mach number flows. in ECCOMAS computational fluid dynamics conference. 1996.

[21] Ferreira, C., Gonzalez, A. et all, "Task 3.2:Development of aerodynamic codes for modelling of flow devices on aerofoils and rotors", EU FP7-Energy-2013-1 / n 608396 AVATAR project deliverable, ECN, Petten, the Netherlands.

[22] Ceyhan, O. "Towards 20MW Wind Turbine: High Reynolds Number Effects on Rotor Design", AIAA-2012-1157, 50th AIAA Aerospace Sciences Meeting, 09 - 12 January 2012, Nashville, Tennessee

[23] Ceyhan Yilmaz, O, Pires O, Manolesos M, Ferreira C. S., "Blind Test Campaign” EU FP7-Energy-2013-1 / n 608396 AVATAR project deliverable, ECN, Petten, the Netherlands.

[24] van Ingen J.L., "The $\mathrm{e}^{\mathrm{N}}$ method for transition prediction. Historical review of work at TU Delft.", AIAA 2008-3830, 38th Fluid Dynamics Conference and Exhibit, 23-26 June 2008, Seattle, Washington.

[25] Rhie, C.M. and Chow, W.L., A numerical study of the turbulent flow past an isolated airfoil with trailing edge separation. AIAA journal, 1983, 21: p. 1525-1532 